\title{
Efeito de um Novo Acelerador de Vulcanização nas Propriedades Reométricas de Composições de Borracha Nitrílica com Diferentes Teores de AN
}

\author{
Iara T. D. de Oliveira, Élen B. A. V. Pacheco, Leila L. Y. Visconte \\ Instituto de Macromoléculas Professora Eloisa Mano, UFRJ
}

\author{
Marcelo R. L. Oliveira, Mayura M. M. Rubinger \\ Departamento de Química, UFV
}

\begin{abstract}
Resumo: O efeito do tipo de acelerador no processo de vulcanização de compostos de borracha nitrílica com dois teores de acrilonitrila foi investigado. Dois aceleradores comerciais, dissulfeto de 2,2'-dibenzotiazol (MBTS) e bis (dimetilditiocarbamato) de zinco (ZDMC), de grupos químicos diferentes, tiazol e ditiocarbamato, respectivamente, foram selecionados e comparados com o composto bis (4 metilfenilsulfonilditiocarbimato-)zincato (II) de tetrabutilamônio (ZNIBU). Os resultados mostram que o tipo de acelerador não só afeta as características de cura, como também tem grande influência nas propriedades físicas. Entre os aceleradores estudados, MBTS foi o menos adequado para a formulação utilizada. Surpreendentemente, verificou-se que o ZNIBU mostrou comportamento intermediário, sendo mais eficaz do que o MBTS e menos eficiente que o ZDMC, considerado um ultra-acelerador, em relação ao tempo ótimo de vulcanização.
\end{abstract}

Palavras-chave: Ditiocarbimato, complexos de zinco, borracha nitrílica, vulcanização.

\section{Effect of a New Accelerator of Vulcanization in the Rheometric Properties of Nitrile Rubber Compositions with Different AN amounts}

\begin{abstract}
The effect of the type of accelerator on the vulcanization process of nitrile rubber compounds with two acrilonitrile amounts was investigated. Two commercial accelerators benzothiazole (MBTS) and zinc dimethyldithiocarbamate (ZDMC), from different chemical groups, thiazole and dithiocarbamate, respectively, were selected and compared with the compound bis (4 methylphenylsulfonyldithiocarbimato-)zincate (II) of tetrabutylammoniun (ZNIBU). The results show that the type of accelerator not only affects the cure characteristics, but also has great influence on the physical properties. Among the accelerators studied, MBTS was found to be the least adequate for the formulation used as far as rheometric parameters are concerned. Surprisingly, it was found that ZNIBU showed an intermediate behavior, being more efficient than MBTS but not as much as ZDMC, considered an ultra accelerator, as for the optimum cure time.
\end{abstract}

Keywords: Dithiocarbimates, zinc complexes, nitrile rubber, vulcanization.

\section{Introdução}

A borracha nitrílica (NBR), devido à sua elevada polaridade, tem excelente resistência a óleo e solventes, baixa permeabilidade a gases e degrada em temperaturas muito elevadas. Por causa dessas características, é amplamente utilizada em produtos como selos de óleo, linhas e mangueiras de combustível ${ }^{[1]}$.

A vulcanização da borracha nitrílica é realizada por meio dos mesmos sistemas usados para vulcanizar as demais borrachas diênicas. De acordo com Fath ${ }^{[2]}$, o sistema enxofre - acelerador é o mais indicado, desde que atenda aos requisitos de cura, de processamento e às propriedades finais do produto. No entanto, outros sistemas como peróxido de dicumila e resina fenólica também são utilizados. A vulcanização com resina fenólica proporciona excelente resistência ao calor, à compressão e boas propriedades dinâmicas ${ }^{[3]}$. Além do sistema de vulcanização, outros aditivos são utilizados na composição de borrachas diênicas, como ativadores, retardantes e inibidores da pré-vulcanização ${ }^{[4]}$. Um típico sistema de vulcanização pode conter entre 0,5 e 4,0 phr de enxofre ou doador de enxofre, um ou mais tipos de aceleradores, de 2 a 10 phr de óxido de zinco e de 1 a 4 phr de ácido esteárico. A relação entre as quantidades de enxofre e acelerador determina os tipos de ligações cruzadas que serão formadas ${ }^{[5]}$.

Sais e complexos de ditiocarbamato são compostos bastante conhecidos e sua ampla aplicabilidade está bem documentada na literatura $^{[6-10]}$. O uso desses complexos metálicos no processo de vulcanização da borracha é um exemplo da sua elevada importância comercial.

Nosso interesse nos ditiocarbimatos deve-se às semelhanças estruturais entre esses compostos e os ditiocarbamatos, os quais são conhecidos por fornecer propriedades interessantes aos compostos de borracha. Em estudos anteriores o bis (4 metilfenilsulfonilditiocarbimato-)zincato (II) de tetrabutilamônio, ZNIBU, foi avaliado como acelerador para composições de NR, mas as taxas de vulcanização observadas com este composto foram mais baixas que as encontradas na presença dos aceleradores comerciais investigados ${ }^{[11,12]}$.

Como compostos contendo ânions ditiocarbimato são pouco estudados e mostraram potencial de vulcanização, foi decidido estender o estudo do ZNIBU a composições de borracha nitrílica, mais polar que a NR, contendo diferentes teores de acrilonitrila, e comparar sua atividade aceleradora de vulcanização com a dos aceleradores comerciais dissulfeto de 2,2-benzotiazol (MBTS) e dimetilditiocarbamato de zinco II (ZDMC).

Autor para correspondência: Leila L. Y. Visconte, Instituto de Macromoléculas Professora Eloísa Mano, Universidade Federal do Rio de Janeiro, 


\section{Experimental}

\section{Materiais e métodos}

Dois tipos de borracha nitrílica, cedidas pela Nitriflex S.A. Indústria e Comércio, foram utilizados: N615B com teor em acrilonitrila (AN) de 31-34\%, viscosidade Mooney (ML $1+4 @ 100{ }^{\circ} \mathrm{C}$ ) 42-52, teor de umidade (\%) 0,0-0,7, teor de cinzas (\%) 0,0-1,0; e tipo N726 com teor em AN de 27-29\%, viscosidade Mooney (ML $\left.1+4 @ 100{ }^{\circ} \mathrm{C}\right) 52-64$, teor de umidade $(\%)$ 0,0-0,7, teor de cinzas (\%) 0,0-1,0.

O composto ZNIBU foi sintetizado segundo descrição da literatura $^{[9]}$ e sua estrutura é apresentada na Figura 1.

\section{Vulcanização}

As borrachas foram misturadas de acordo com a norma ASTM D 3187, seguindo as formulações apresentadas na Tabela 1. Como pode ser observado o teor e tipo de acelerador, bem como o conteúdo em acrilonitrila da borracha foram alterados, permanecendo os demais aditivos constantes. Os aditivos foram utilizados conforme recebidos e as composições foram preparadas em um misturador aberto de rolos, processadas em temperatura ambiente e com taxa de fricção $1: 1,25$. Os parâmetros reométricos foram determinados em um reômetro de disco oscilatório (ODR), da marca Tecnologia Industrial, operando a $150{ }^{\circ} \mathrm{C} \mathrm{e} 1^{\circ}$ de arco, de acordo com a norma ASTM D 2084. As misturas foram vulcanizadas em uma prensa hidráulica a $150{ }^{\circ} \mathrm{C}$ e $3,0 \mathrm{MPa}$. A partir do material vulcanizado foram obtidos os corpos de prova para os testes de densidade de ligação cruzada e deformação permanente a compressão (DPC), determinados segundo a Equação de Flory-Rehner (Equação 1) e a norma ASTM D 395-85, respectivamente.

$$
2-p-\mathrm{CH}_{3} \mathrm{C}_{6} \mathrm{H}_{4} \mathrm{SO}_{2} \mathrm{~N}=\mathrm{CS}_{2} \mathrm{~K}_{2} \cdot 2 \mathrm{H}_{2} \mathrm{O}+\mathrm{Zn}\left(\mathrm{CH}_{3} \mathrm{COO}\right)_{2} \cdot 2 \mathrm{H}_{2} \mathrm{O}+2 \mathrm{Bu}_{4} \mathrm{NBr}
$$

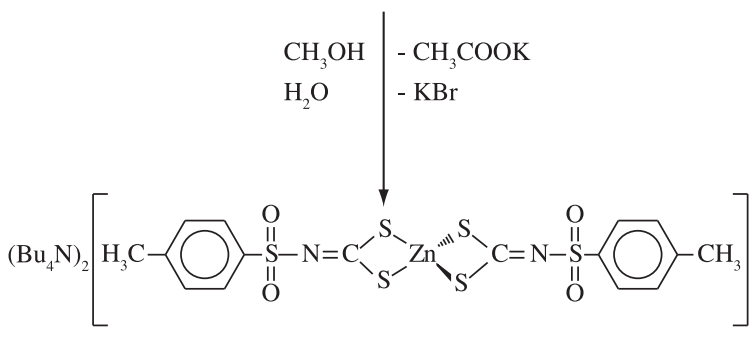

ZNIBU

Figura 1. Esquema de preparação do ZNIBU.

$$
\rho=\frac{-\left\{\ln \left(1-V_{r}\right)+V_{r}+X \cdot V_{r}^{2}\right\}}{\left\{V_{0}\left(V_{r}^{1 / 3}-V_{r} / 2\right)\right\}}
$$

Onde $V_{r}$ é a fração em volume da borracha na rede inchada, dada pela razão volume inicial/volume do inchado; $X$ é o parâmetro de interação entre a borracha e o solvente; $V_{0}$ é o volume molar do solvente $\left(\mathrm{cm}^{3} / \mathrm{g} \cdot \mathrm{mol}\right)$. O termo $V_{r}$ depende da capacidade do solvente de inchar a borracha, isto é, quanto mais alta capacidade de inchamento, tanto mais baixo será $V_{r}$.

$\mathrm{O}$ valor de $V_{r}$ depende também da densidade de ligações cruzadas. Densidade de ligações cruzadas mais altas gera maior concentração de reticulação, resultando em menor inchamento, isto é, mais alto valor de $V_{r}$ para o mesmo solvente ${ }^{[13,14]}$.

Neste trabalho o solvente escolhido foi a acetona, com volume molar $V_{0}=73,4 \mathrm{~cm}^{3} / \mathrm{g}$.mol. Como parâmetro de interação NBR-acetona foi usado o valor 0,345 que é o mesmo para os dois tipos de elastômeros nitrílicos, cujos parâmetros de solubilidade são também similares, $\mathrm{d}=9,36(\mathrm{cal} / \mathrm{cm})^{2[13-18]}$. $\mathrm{O}$ inchamento no equilíbrio foi atingido após 7 dias, sob temperatura ambiente e na ausência de luz. O ensaio foi feito em triplicata para cada composição em amostras de dimensões de 2,0 $\times 2,0 \times 0,2 \mathrm{~cm}$.

Os testes de deformação permanente à compressão (DPC) foram realizados de acordo com a norma ASTM D 395-85. Os corpos de prova foram vulcanizados em molde adequado para o ensaio, em triplicata. A temperatura de realização do teste foi de $70{ }^{\circ} \mathrm{C}$ e os corpos foram submetidos à compressão no período de 22 horas. $\mathrm{O}$ ensaio realizado usando-se espaçadores metálicos e o resultado foi baseado na média dos valores obtidos.

\section{Resultados e Discussão}

Tradicionalmente, nas formulações de borracha as quantidades dos componentes são dadas em phr ("parts per hundred of resin"). Em trabalhos anteriores ${ }^{[12]}$ este método tradicional foi seguido e foi observado que, em composições de borracha natural, a ação aceleradora do ZNIBU ficou aquém do desejável. Uma das justificativas levantadas para esta observação baseou-se na diferença em polaridade entre o acelerador e a borracha utilizada. No entanto, comparando-se as massas molares dos diferentes aceleradores, temos os seguintes valores: $\mathrm{ZNIBU}=1012,96$ u.m.a; $\mathrm{ZDMC}=305,84$ u.m.a e MBTS $=332,48$ u.m.a. Surge então a possibilidade de se ter usado naquela oportunidade, quantidade de ZNIBU insuficiente para assegurar velocidades de vulcanização comparáveis às conferidas pelos aceleradores comerciais. Neste trabalho optou-se, então, por utilizar quantidades molares dos aceleradores, enquanto para os demais aditivos as quantidades foram mantidas em phr.

\begin{tabular}{|c|c|c|c|c|c|c|c|}
\hline \multirow[t]{2}{*}{ Borracha } & \multirow[t]{2}{*}{ Composição } & \multirow[t]{2}{*}{ Acelerador } & \multicolumn{2}{|c|}{ Massa de acelerador (g) } & \multirow{2}{*}{$\begin{array}{l}\text { Ácido esteárico } \\
\text { (phr) }\end{array}$} & \multirow{2}{*}{$\begin{array}{c}\text { Óxido de zinco } \\
\text { (phr) }\end{array}$} & \multirow{2}{*}{$\begin{array}{c}\text { Enxofre } \\
\text { (phr) }\end{array}$} \\
\hline & & & em $0,8 \times 10^{-3} \mathrm{~mol}$ & em $1,2 \times 10^{-3} \mathrm{~mol}$ & & & \\
\hline \multirow{6}{*}{ 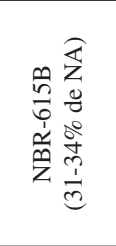 } & INI601 & \multirow{2}{*}{ ZNIBU } & 0,81 & - & \multirow{6}{*}{1,0} & \multirow{6}{*}{3,0} & \multirow{6}{*}{1,5} \\
\hline & INI611 & & - & 1,21 & & & \\
\hline & INM601 & \multirow{2}{*}{ ZDMC } & 0,24 & - & & & \\
\hline & INM611 & & - & 0,36 & & & \\
\hline & INB601 & \multirow{2}{*}{ MBTS } & 0,26 & - & & & \\
\hline & INB611 & & - & 0,39 & & & \\
\hline \multirow{6}{*}{ 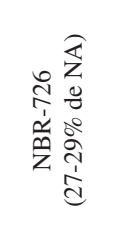 } & TNI701 & \multirow{2}{*}{ ZNIBU } & 0,81 & - & \multirow{6}{*}{1,0} & \multirow{6}{*}{3,0} & \multirow{6}{*}{1,5} \\
\hline & TNI711 & & - & 1,21 & & & \\
\hline & TNM701 & \multirow{2}{*}{ ZDMC } & 0,24 & - & & & \\
\hline & TNM711 & & - & 0,36 & & & \\
\hline & TNB701 & \multirow{2}{*}{ MBTS } & 0,26 & - & & & \\
\hline & TNB711 & & - & 0,39 & & & \\
\hline
\end{tabular}

Tabela 1. Formulação dos compostos de borracha. 
Com o objetivo de investigar a influência do tipo e da quantidade de acelerador e do teor de acrilonitrila sobre a vulcanização das diferentes composições de NBR, as misturas foram caracterizadas por meio de curvas de torque versus tempo, obtidas em ODR, como mostram as Figuras 2 e 3. De cada uma dessas curvas parâmetros como torque mínimo, torque máximo, $\mathrm{t}_{\mathrm{s} 1}$ e $\mathrm{t}_{90}$ foram extraídos $\mathrm{e}$ estão apresentados na Tabela 2.

Ao serem analisados os tempos ótimos de vulcanização $\left(t_{90}\right)$ para ambas as borrachas, observa-se que os valores fornecidos pelo ZNIBU situam-se entre aqueles obtidos com o MBTS e aqueles dados pelo ZDMC. Esta tendência se mantém independentemente

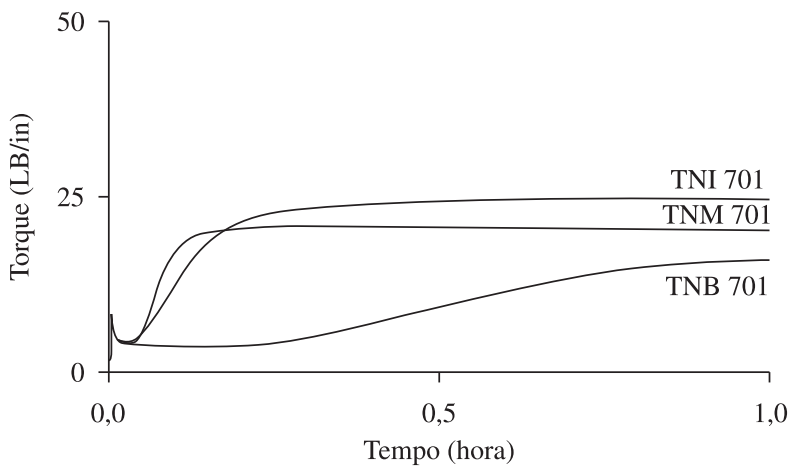

(a) da quantidade de acelerador, embora a vulcanização seja mais rápida, isto é, $\mathrm{t}_{90}$ é menor, quando maior o teor de acelerador presente.

Considerando-se cada uma das borrachas nitrílicas, o tempo de pré-cura (tempo disponível antes da cura propriamente dita), das misturas contendo ZNIBU e ZDMC não sofreu alteração em função do aumento no teor desses aceleradores. Já o aumento no teor de MBTS provocou uma redução significativa em $t_{\mathrm{sl}}$, no caso da NBR 615B. As propriedades reométricas fornecidas pelas composições com a NBR 726 foram menos sensíveis à variação na quantidade dos aceleradores.

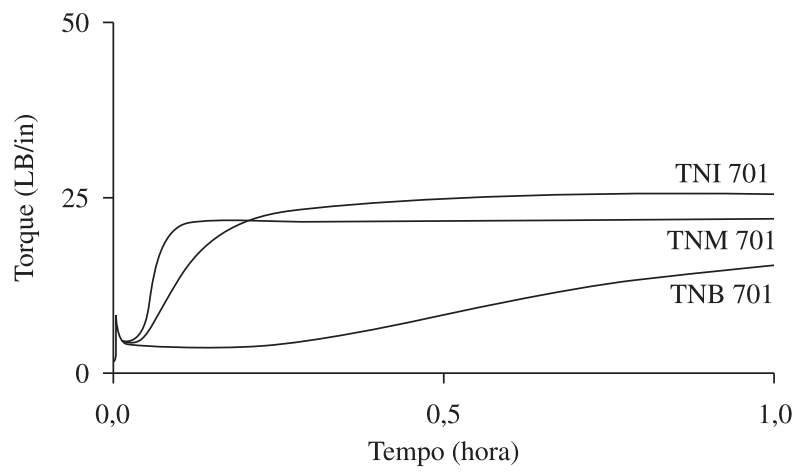

(b)

Figura 2. Curvas reométricas das composições de NBR 726 vulcanizadas com diferentes aceleradores em quantidades equivalentes a: a) $0,8 \times 10^{-3}$ mol; e b) $1,2 \times 10^{-3} \mathrm{~mol}$.

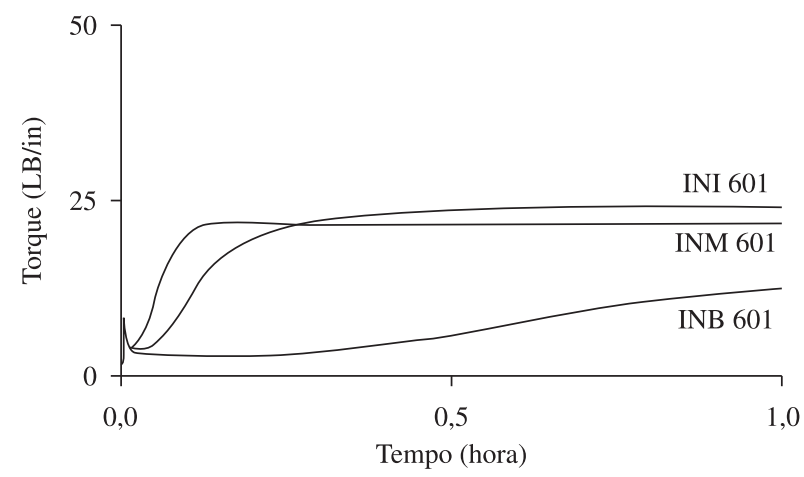

(a)

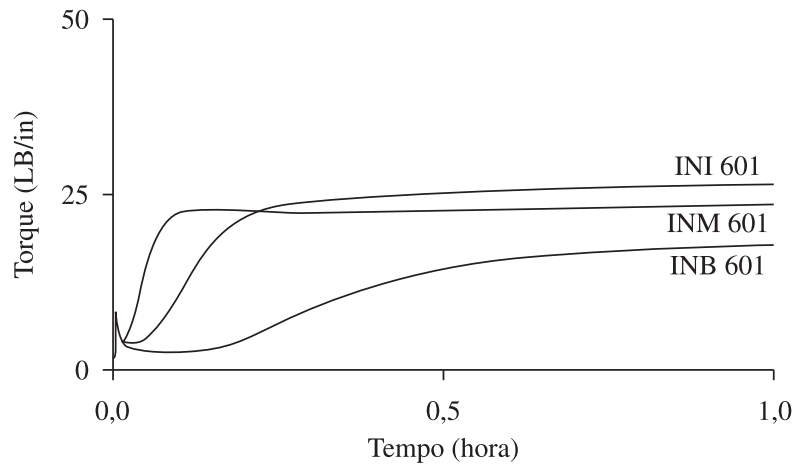

(b)

Figura 3. Curvas reométricas das composições de NBR $615 \mathrm{~B}$ vulcanizadas com diferentes aceleradores em quantidades equivalentes a: a) $0,8 \times 10^{-3}$ mol; e b) $1,2 \times 10^{-3} \mathrm{~mol}$

Tabela 2. Parâmetros reométricos obtidos a $150^{\circ} \mathrm{C}$ de misturas de NBR contendo ZNIBU, ZDMC ou MBTS como acelerador de vulcanização.

\begin{tabular}{|c|c|c|c|c|c|c|c|c|}
\hline Borracha & Composição & Acelerador & $\begin{array}{c}\mathbf{M}_{L} \\
(\mathbf{L b} / \mathbf{i n})\end{array}$ & $\begin{array}{c}\mathbf{M}_{\mathrm{H}} \\
(\mathbf{L b} / \mathbf{i n})\end{array}$ & $\begin{array}{c}\Delta \\
(\mathbf{L b} / \mathrm{in})\end{array}$ & $\begin{array}{c}\mathbf{t}_{\mathrm{s1}} \\
\text { (minutes) }\end{array}$ & $\begin{array}{c}\mathbf{t}_{90} \\
\text { (minutes) }\end{array}$ & CRI \\
\hline \multirow{6}{*}{ 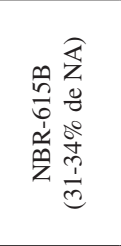 } & INI601 & \multirow{2}{*}{ ZNIBU } & 3,3 & 23,2 & 19,9 & 3 & 17,4 & 6,9 \\
\hline & INI611 & & 3,6 & 23,8 & 20,2 & 3 & 15 & 8,3 \\
\hline & INM601 & \multirow{2}{*}{ ZDMC } & 3,7 & 21,2 & 17,5 & 1,2 & 5,4 & 23,8 \\
\hline & INM611 & & 4,6 & 21,5 & 16,9 & 1,2 & 4,8 & 27,7 \\
\hline & INB601 & \multirow{2}{*}{ MBTS } & 2,6 & 10 & 7,4 & 23,4 & 55,2 & 3,1 \\
\hline & INB611 & & 2,6 & 15,8 & 13,2 & 10,8 & 47,8 & 3,7 \\
\hline \multirow{6}{*}{ 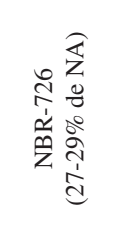 } & TNI701 & \multirow{2}{*}{ ZNIBU } & 4,3 & 25 & 20,7 & 3 & 16,2 & 8,3 \\
\hline & TNI711 & & 4,5 & 25,1 & 20,6 & 3 & 15 & 7,5 \\
\hline & TNM701 & \multirow{2}{*}{ ZDMC } & 3,9 & 21,1 & 17,2 & 2,4 & 7,2 & 20,8 \\
\hline & TNM711 & & 4,1 & 21,9 & 17,8 & 2,4 & 5,4 & 33,3 \\
\hline & TNB701 & \multirow{2}{*}{ MBTS } & 3,6 & 14,8 & 11,2 & 18 & 51,6 & 2,9 \\
\hline & TNB711 & & 3,6 & 16,4 & 12,8 & 17,4 & 49,2 & 3,1 \\
\hline
\end{tabular}

$\mathrm{M}_{\mathrm{L}}=$ torque mínimo, $\mathrm{M}_{\mathrm{H}}=$ torque máximo, $\Delta \mathrm{M}=\mathrm{M}_{\mathrm{H}}-\mathrm{M}_{\mathrm{L}}, \mathrm{t}_{\mathrm{s} 1}=$ tempo de pré-cura ou $\operatorname{scorch}, \mathrm{t}_{90}=$ tempo a $90 \%$ de cura (tempo ótimo de cura), CRI = índice de velocidade de cura. 
O torque mínimo está diretamente relacionado à processabilidade das composições não vulcanizadas. Pode-se observar, a partir ainda da Tabela 2, que melhor processabilidade é conseguida com o MBTS como acelerador, independente do teor usado e da borracha. Em baixos teores de AN, o ZNIBU é o acelerador que confere a pior processabilidade (os mais altos valores de $t_{s 1}$ ). Essa tendência inverte-se quando a borracha apresenta teores mais elevados de AN.

Nesta mesma tabela pode-se verificar que os valores de torque máximo são maiores para as composições vulcanizadas com ZNIBU, enquanto os menores valores foram encontrados para as composições com MBTS, para ambas as borrachas.

A diferença entre os torques máximo e mínimo está relacionada com o grau de cura e valores mais elevados foram encontrados para ZNIBU e ZDMC, sugerindo que estes dois compostos foram os aceleradores mais eficientes na formação de ligações cruzadas. Dentre esses dois compostos, o ZNIBU mostrou-se ainda ligeiramente superior ao ZDMC.

A observação dos parâmetros reométricos com os gráficos das Figuras 2 e 3 mostram que, dentre os três aceleradores investigados, o ZDMC é o que apresenta a mais alta taxa de vulcanização. No entanto, uma veziniciada, a vulcanização comZNIBU é mais eficiente na formação das ligações. Observa-se também que o aumento na concentração molar desses aceleradores teve influência pouco significativa sobre os parâmetros, diferentemente ao observado com o composto MBTS. Este acelerador apresentou comparativamente o pior resultado para a vulcanização dos compostos de NBR. Tabela 3 apresenta os resultados de deformação permanente por compressão (DPC).

Os ensaios de deformação permanente à compressão (DPC) são realizados com o objetivo de aferir a habilidade das composições de borracha em manter propriedades elásticas após ação de uma força compressiva. Quanto maior o valor de DPC, menor tende a ser a capacidade do material elastomérico em manter suas propriedades elásticas. Durante esse ensaio as amostras são expostas a uma temperatura de $70{ }^{\circ} \mathrm{C}$ ao longo de 22 horas, o que pode levar a uma leve degradação térmica, que acarretaria em perdas nas propriedades elásticas.

Observa-se que a adição crescente dos diferentes tipos de acelerador às misturas apresentou resultados compatíveis com dados da literatura. $\mathrm{O}$ aumento da concentração molar dos aceleradores levou à redução no valor de DPC. À exceção das composições vulcanizadas com ZDMC, os valores encontrados foram maiores quando a NBR-615B (maiores teores de AN) foi usada.

\section{Densidade de ligação cruzada}

Todos os elastômeros absorvem líquidos e esta absorção provoca um aumento no volume da borracha ou "inchamento". As borrachas, antes da vulcanização, são totalmente solúveis em determinados solventes, diferentemente das borrachas vulcanizadas que são insolúveis. As ligações cruzadas químicas são ligações fortes entre as cadeias dos elastômeros, que impedem a completa dispersão das moléculas dos mesmos no solvente e, desta forma, restringem a deformação do elastômero ${ }^{[19,20]}$.

A Tabela 4 apresenta os valores de densidade de ligações cruzadas para as composições de borracha nitrílica.

A partir da Tabela 4 e com base na técnica do inchamento no equilíbrio, pode-se verificar que, de um modo geral, os valores de $V_{r}$ são sempre crescentes com o aumento do teor de acelerador utilizado. Isso ocorre provavelmente devido ao aumento das ligações mono e dissulfídicas formadas. As ligações mono e dissulfídicas são menores que as ligações polissulfídicas o que dificulta a difusão do solvente entre as cadeias elastoméricas reduzindo o inchamento. Pode-se constatar ainda que o acelerador ZNIBU apresentou os maiores
Tabela 3. Valores de deformação permanente a compressão.

\begin{tabular}{ccccc}
\hline Acelerador & Amostra & $\begin{array}{c}\text { Massa (g) em } \\
\mathbf{0 , 8} \times \mathbf{1 0}^{-3} \mathbf{~ m o l}\end{array}$ & $\begin{array}{c}\text { Massa (g) em } \\
\mathbf{1 , 2} \times \mathbf{1 0}^{-3} \mathbf{~ m o l}\end{array}$ & $\begin{array}{c}\text { DPC } \\
(\mathbf{\%})\end{array}$ \\
\hline \multirow{2}{*}{ ZNIBU } & INI 601 & 0,81 & - & 53,5 \\
& INI 611 & - & 1.21 & 47,8 \\
\multirow{2}{*}{ ZDMC } & INM 601 & 0,24 & - & 28,1 \\
& INM 611 & - & 0,36 & 25,8 \\
\multirow{2}{*}{ MBTS } & INB 601 & 0,26 & - & 65,2 \\
& INB 611 & - & 0,39 & 51,9 \\
\hline \multirow{2}{*}{ ZNIBU } & TNI 701 & 0,81 & - & 46,5 \\
& TNI 711 & - & 1.21 & 35,6 \\
\multirow{2}{*}{ ZDMC } & TNM 701 & 0,24 & - & 43.3 \\
& TNM 711 & - & 0,36 & 36,6 \\
\multirow{2}{*}{ MBTS } & TNB 701 & 0,26 & - & 27,6 \\
& TNB 711 & - & 0,39 & 27,5 \\
\hline
\end{tabular}

Tabela 4. Densidade de ligações cruzadas das composições de NBR.

\begin{tabular}{ccccc}
\hline Amostra & $\begin{array}{c}\text { Massa (g) em } \\
\mathbf{0 , 8} \times \mathbf{1 0}^{-3} \mathbf{~ m o l}\end{array}$ & $\begin{array}{c}\text { Massa (g) em } \\
\mathbf{1 , 2} \times \mathbf{1 0}^{-3} \mathbf{~ m o l}\end{array}$ & $\begin{array}{c}\rho\left(\mathbf{m o l . c m}^{-3}\right) \\
\left(\times \mathbf{1 0}^{-4}\right)\end{array}$ & $\begin{array}{c}\text { Desvio } \\
\text { padrão }\end{array}$ \\
\hline INI 601 & 0,81 & - & 38,0 & 0,02 \\
INI 611 & - & 1,21 & 38,2 & 0,00 \\
INM 601 & 0,24 & - & 37,5 & 0,07 \\
INM 611 & - & 0,36 & 38,5 & 0,00 \\
INB 601 & 0,26 & - & 13,9 & 0,64 \\
INB 611 & - & 0,39 & 27,0 & 0,94 \\
\hline TNI 701 & 0,81 & - & 36,3 & 0,08 \\
TNI 711 & - & 1,21 & 37,2 & 0,18 \\
TNM 701 & 0,24 & - & 37,8 & 0,29 \\
TNM 711 & - & 0,36 & 37,9 & 0,27 \\
TNB 701 & 0,26 & - & 36,7 & 0,20 \\
TNB 711 & - & 0,39 & 37,6 & 0,17 \\
\hline
\end{tabular}

valores de densidade de ligação cruzada, quando comparado ao ZDMC e MBTS, nas composições com a NBR-615B. Os resultados de densidade de ligação cruzada estão de acordo com as diferenças entre torques máximo e mínimo obtidos do reômetro de disco oscilatório, e, mais uma vez, mostram o potencial de vulcanização do ZNIBU.

\section{Conclusão}

Os ensaios reométricos mostraram que as composições de NBR vulcanizadas com o composto ZNIBU como acelerador apresentaram valores de $\Delta \mathrm{M}$ próximos aos obtidos com o acelerador comercial ZDMC. Os valores fornecidos por esses dois aceleradores foram mais elevados que aqueles fornecidos pelo MBTS, sugerindo que este último não é tão eficiente na cura de composições de NBR quanto os sais de zinco estudados. $\mathrm{O}$ valor de $\mathrm{M}_{\mathrm{H}}$ medido a pequenas deformações, é normalmente proporcional à quantidade de ligações cruzadas formadas por volume de borracha, isto é, à densidade de ligações cruzadas. Os testes de densidade de ligação cruzada corroboraram os resultados obtidos pela análise reométrica, aumentando conforme o aumento do teor de acelerador, indicando aumento na formação de ligações mono e dissulfidicas.

\section{Agradecimentos}

Os autores agradecem a Nitriflex. S.A, ao CNPq, Capes e Faperj pelo apoio concedido. 


\section{Referências Bibliográficas}

1. Shiny, P. et al. - J. Elast. and Plast., 38, p.199 (2006).

2. Fath. A. M. et al. - R. Chem. Stab., 35, p.490 (1982).

3. Sousa, A. M. F. et al. - J. Ap. Polym. Sci., 84, p.505 (2002).

4. Morton, M. R. - "Techny”, Krieger Publishing Company, Florida (1981).

5. Celestino, M. L. - "Desenvolvimento de misturas elastoméricas de borracha acrílica e borracha nitrílica", Dissertação Mestrado, Universidade Federal do Rio de Janeiro, Instituto de Macromoléculas Professora Eloisa Mano, Brasil (2007).

6. Manohar, A. et al. - Inorg. Chim. Acta, 314, p.177 (2001).

7. Oliveira, M. R. L. et al. - Trans. Metal. Chem., 28, p.455 (2003).

8. Oliveira, M. R. L. et al.-. Polyh, 23, p.1153 (2004).

9. Faria Franca, E. et al. - Polyh, 25, p.2119 (2006).

10. Amim, R. S. et al. - Polyh, 27, p.1891 (2008).

11. Hofmann, W. - "Rubber Technology Handbook", Hanser, New York (1989).
12. Mariano, R. M. et al. - Europ. Polym. J., 43, p.4706 (2007).

13. Flory, P. J. \& Rehner, J. J. J. - Chem. Phys., 11, p.521 (1943).

14. Boonstra, B. B. - Polym., 20, p.691 (1979).

15. Brundrup, J. - "Polymer Handbook", $4^{\text {th }}$ ed, p.154.

16. Pethrik, R. A. - Polymr. Yearb., Harwood Academic Publishers, NY, (1985)

17. Orwell, R. A. - R. Chem. Tech., 50, p.451 (1977).

18. Fedors, R. F. - Polymr. Eng. Sci., 14, p.147 (1974).

19. Harwood, J. A. C. - R. Techn. Manuf., Newnes-Butterworths, London, cap. 3, p.56-76 (1982).

20. Lee, S. - R. Chem. Tech., 67, p.854 (1994).

Enviado: 07/02/10

Reenviado: $17 / 06 / 10$

Aceito: $18 / 06 / 10$

DOI: $10.1590 / \mathrm{S} 0104-14282010005000059$ 